\title{
Clustering-Based Mobile Gateway Management in Integrated CRAHN-Cloud Network
}

\author{
Ling Hou ${ }^{1}$, Angus K.Y. Wong ${ }^{1}$, Alan K. H. Yeung ${ }^{2}$, Steven S. O. Choy ${ }^{1}$ \\ ${ }^{1}$ School of Science and Technology, The Open University of Hong Kong \\ Ho Man Tin, Kowloon, Hong Kong \\ [e-mail: lhou@ouhk.edu.hk, akywong@ouhk.edu.hk, sochoy@ouhk.edu.hk ] \\ ${ }^{2}$ Department of Electronic Engineering, City University of Hong Kong \\ Kowloon Tong, Kowloon, Hong Kong \\ [e-mail: eeayeung@cityu.edu.hk] \\ *Corresponding author: Angus K.Y. Wong
}

Received May 7, 2017; revised October 11, 2017; revised January 10, 2018; accepted February 5, 2018; published July 31, 2018

\begin{abstract}
The limited storage and computing capacity hinder the development of cognitive radio ad hoc networks (CRAHNs). To solve the problem, a new paradigm of cloud-based CRAHN has been proposed, in which a CRAHN will make use of the computation and storage resources of the cloud. This paper envisions an integrated CRAHN-cloud network architecture. In this architecture, some cognitive radio users (CUs) who satisfy the required metrics could perform as mobile gateway candidates to connect other ordinary CUs with the cloud. These mobile gateway candidates are dynamically clustered according to different related metrics. Cluster head and time-to-live value are determined in each cluster. In this paper, the gateway advertisement and discovery issues are first addressed to propose a hybrid gateway discovery mechanism. After that, a QoS-based gateway selection algorithm is proposed for each CU to select the optimal gateway. Simulations are carried out to evaluate the performance of the overall scheme, which incorporates the proposed clustering and gateway selection algorithms. The results show that the proposed scheme can achieve about $11 \%$ higher average throughput, $10 \%$ lower end-to-end delay, and $8 \%$ lower packet drop fractions compared with the existing scheme.
\end{abstract}

Keywords: cognitive radio ad hoc networks, mobile gateway, cloud computing, clustering

The work described in this paper was fully supported by a grant from the Research Grants Council of the Hong Kong Special Administrative Region, China (UGC/FDS16/E05/14). 


\section{Introduction}

Cognitive radio (CR) is a promising technology to alleviate the spectrum scarcity problem [1-3]. Cognitive radio networks (CRNs), equipped with the intrinsic capabilities of cognitive radios, can provide the capability to share the spectrum with primary users (PUs), i.e., the legitimate users of the spectrum, in an opportunistic manner [4, 5]. PUs could use allocated spectrum channels whenever there is information to be transmitted. Instead, CUs should sense and utilize available spectrum channels (i.e., those channels temporally unused by their primary users), and it has to immediately vacate this channel as soon as the PU uses it again. Cognitive radio ad hoc network (CRAHN) is a kind of CRN formed in a distributed multi-hop manner. CRAHNs have many applications, like military applications, vehicular surveillance, and disaster relief $[6,7]$.

There is a significant challenge that hinders the development of CRAHNs. CR devices in CRAHNs are typically equipped with limited storage, computing and battery capacities. However, as mentioned earlier, CUs continuously detect, utilize, and vacate spectrum channels, which are inherently computationally intensive and have already consumed certain recourse of the CR devices. As a result, $\mathrm{CR}$ devices do not have much remaining resource to perform sophisticated functions [8, 9]. Recently, a new paradigm of cloud-based CRNs, which is an integration of CRNs with the cloud, has been gaining a great deal of attention [9-13]. Cloud computing, with supply of computing and storage resources on demand, is widely recognized as next generation computing paradigm [14-16]. In [17], a hierarchical cloud computing architecture is proposed to enhance performance by adding a mobile cloud formed by mobile devices to a static cloud. It gives insights into the architecture for integrating CRNs with the cloud. The integration of CRNs and the cloud allows CUs in CRNs to use the computation and storage resources of the cloud. In order to integrate a CRAHN with the cloud, gateways are needed to act as the portals between a CRAHN and the cloud due to their differences in communication protocols. Due to the characteristics of mobility and changing network topology of the CRAHN, mobile gateways [18-21] are introduced to provide stable connections to the cloud for CUs.

\subsection{Purpose of mobile gateways}

For CUs to obtain stable cloud services, it is essential to maintain stable connectivity to the cloud. In most of the existing literature, gateways are considered static, deployed at fixed position. The dynamic and multi-hop nature of CRAHN communication impacts the stability of path to these gateways. Additionally, as these gateways are fixed, the routing and discovery mechanisms are mainly proactive. Though proactive routing mechanisms reduce delay, they increase the signaling overhead and require frequent changes in the pre-defined routing tables of CUs. To cope with these shortcomings, we use mobile gateways in this paper for CRAHN to integrate with the cloud. The CUs in the CRAHN, which satisfy the essential criteria and required metric information, are seen as mobile gateway candidates. The focus of this paper is on designing a scheme for each source to choose a better mobile gateway to act as its portal to the cloud.

With this regard, efficient gateway discovery mechanisms are also required. As proactive gateway discovery reduces delay and reactive discovery reduces signaling overhead, this paper proposes a hybrid gateway discovery mechanism for CRAHN, combining the pros of both proactive and reactive gateway discovery. This requires adequate configuration of 
different parameters such as advertisement zone (i.e., in terms of Time To Live (TTL) value). In this paper, we address all the above-mentioned issues by devising a mobile gateway management mechanism.

\subsection{Purpose of clustering}

CRAHNs have features of 1) dynamic spectrum availability due to PU activities, 2) CU mobility and 3) self-organizing. However, when the number of CUs increases, with each CU handling its own decentralized sensing, routing and neighborhood connectivity maintenance tasks, serious scalability and hidden terminal problems may occur. The most common solution adopted for this problem is clustering [22-25]. Clustering aims to organize CUs into groups, based on some specific common characteristics. For CRAHNs, clustering would be an effective management methodology for a number of reasons. First of all, the accuracy of spectrum sensing could be improved. Coordination of the CUs in the same cluster in sensing could prevent interference originating from CUs and hidden terminal problems. Secondly, the chance of collisions among neighboring CUs is reduced. Hence, the performance of resource utilization could be improved. Finally, clustering enhances the effectiveness of broadcasting and relaying of messages, which simplifies the routing process.

This paper aims at clustering only gateway candidates (to be described later) according to key metrics relevant with topology changes. This paper also aims at selecting, out of each cluster, a cluster head that serves as the default gateway in the cluster to connect CRAHN with the cloud. The reasons of clustering only gateway candidates are given as follows. First, it would reduce the complexity of clustering. Second, it would be more flexible and robust because any other gateway candidate in the cluster could be a backup if the cluster head fails. Finally, it would decrease the consumption of network resources because the flows in a cluster can be multiplexed by one gateway that handles to send these flows to the cloud.

In the area of clustering in cognitive radio networks, there has been a lot of research work, but only a few of them considered the stability of the cluster formed. In [26], Li and Gross proposed two clustering schemes named as ROSSDGA and ROSS-DFA. The schemes increase common channels within a cluster and between clusters to enhance the inter- and intra-cluster connectivity by utilizing 1-hop neighbor knowledge. In [27], Lazos et al. proposed a distributed clustering algorithm based on channel availability. In [28], Lin et al. proposed a clustering algorithm using the Connected-dominating-set (CDS), which could maximize the lifetime of the cluster. Though these works considered the cluster changes due to spectrum availability, they did not consider the cluster changes caused by CU mobility.

The main challenge for clustering in CRAHN lies in the dynamic topology changes as a result of PU activities as well as mobility of CUs. Thus, an efficient clustering to maintain cluster stability should consider the impact of both PU activities and mobility of CUs. Otherwise, the performance will be degraded due to frequent re-clustering operations. Frequent clustering process will not only consume time, but also interrupt the ongoing communications. In this paper, we will take the frequent topology changes into account in the clustering algorithm to prevent unnecessary re-clustering.

The rest of this paper is structured as follows. Section 2 presents the network architecture. Section 3 describes the methodology of clustering and introduces our proposed clustering algorithm. Section 4 presents our mobile gateway management mechanism, including gateway discovery and gateway selection algorithms. The performance of the proposed scheme is evaluated in Section 5. Conclusion is given in Section 6. 


\section{System Model}

The topology of our envisioned integration of CRAHN and cloud is depicted in Fig. 1. The elements involved in the system include the following:

PUs: Each PU possesses a set of licensed channels for the licensed service. The activities of each PU on its licensed channels are modeled as an ON-OFF process, where each channel has two states: ON and OFF. An ON (busy) state represents the period during which the channel is occupied by its PU, while an OFF (idle) state represents the period during which the channel is idle. For simplicity, we assume that each PU has a fixed interference range.

Ordinary CUs: The ordinary CUs do not have a license to operate in a licensed channel. They can only opportunistically access licensed spectrum channels which are in OFF state. Hence, CUs need to perform spectrum sensing to find available spectrum channels. If a CU is located in the interference area of a PU, then the available spectrum channels for this $\mathrm{CU}$ would be affected by the activities of this PU. We use $\left\{\mathbb{P}_{i}\right\}$ to denote all the PUs whose interference area covers $\mathrm{CU} i$. Thus, the available spectrum set of a CU would be affected by the activities of all the PUs in $\left\{\mathbb{P}_{i}\right\}$. In the system, CUs are mobile and can communicate with each other in a multi-hop manner on available spectrum channels. CUs are assumed to be equipped with GPS devices to get their locations and moving velocities in the Cartesian space.

Cloud: A cloud is established among a group of dedicated servers in the Internet. It could supply computing and storage resources on demand.

Gateway Candidates (GWCs): Gateway candidates are a kind of CUs, which could connect to the cloud directly and act as the portal for other CUs to the cloud. Due to the movement of the CUs, it is difficult and costly to provide stable cloud services via stationary gateways. To remedy the limitations of stationary gateways, mobile gateways candidates are applied in this work to improve the cloud services. As given in [9], mobile gateway candidates could be some Multi-input Multi-output (MIMO) radios mounted on vehicles. Each GWC is equipped with a GPS device to determine its location in the Cartesian space.

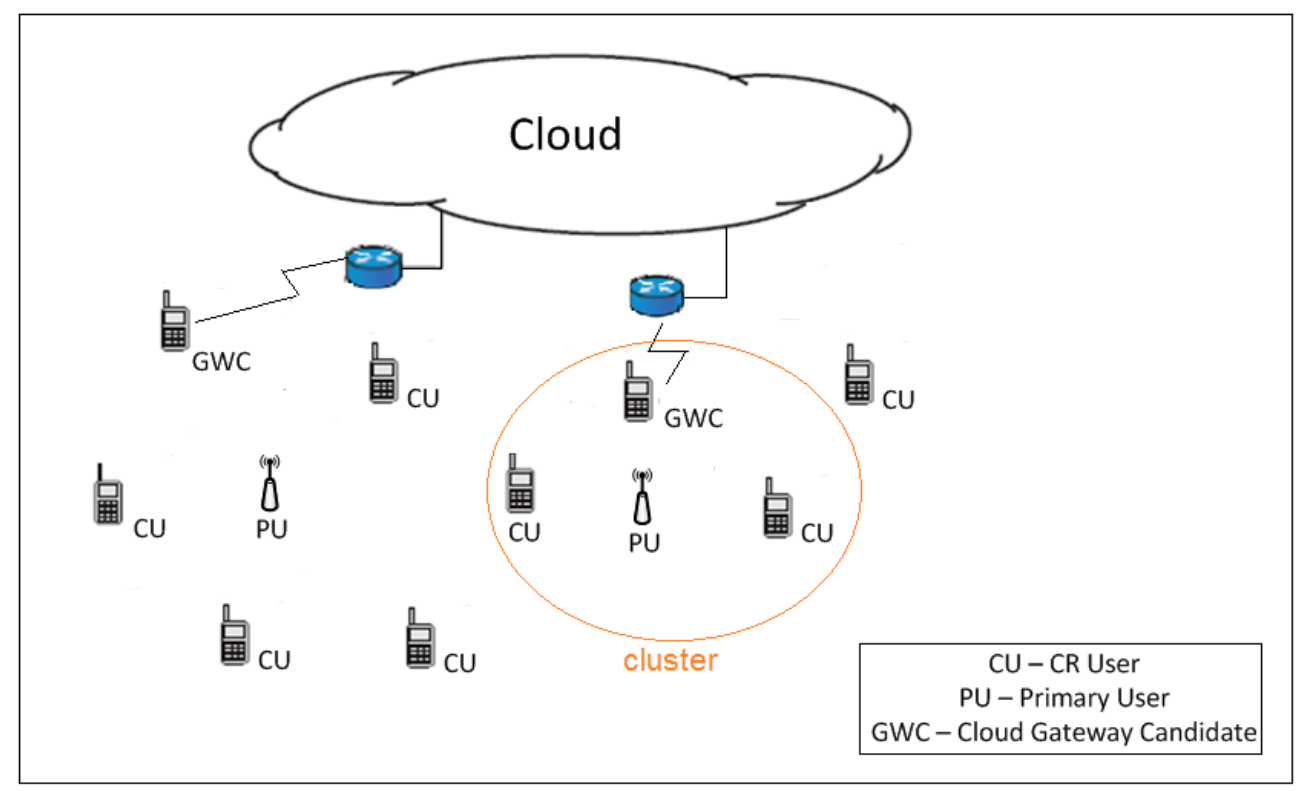

Fig. 1. Integrated CRAHN-Cloud architecture 
The prime objective of the architecture is to provide stable cloud service for CRAHNs. To achieve this objective, we proceed in two stages. Firstly, clustering and $\mathrm{CH}$ selection are discussed and relevant algorithms are given to construct relatively stable network backbone. Secondly, we design a mobile gateway management mechanism, which consists of two functions 1) Hybrid gateway discovery/advertisement, and 2) QoS-based gateway selection.

\section{Multi-Metric Clustering}

As stated earlier, clustering is an effective management methodology for a CRAHN. In this paper, clustering is performed with considering two factors: 1) PU interference range and 2) link stability. Clusters are formed with the gateway candidates only. Ordinary CUs, as shown in Fig. 1, can only select the gateway candidates in these clusters for connection with the cloud.

\subsection{Clustering based on PU interference range}

Recall that PUs can utilize their licensed channels directly, whereas CUs can only opportunistically access available spectrum channels, which are not being used by the PUs. Thus, the available spectrum set of a CU would be affected by the activities of PUs whose interference area covers this CU. Therefore, CUs located in the same PU interference area have more common available channels. For two adjacent CUs to communicate with each other directly, they must have at least one common available channel. It is intuitive that two nodes with more common available channels have a higher probability of constructing robust links. Thus, GWCs located in the same PU interference area should be grouped into a cluster. GWCs could determine their locations in the PU interference area through spectrum sensing.

\subsection{Clustering based on link stability}

The links between CUs are affected by CU mobility as well as PU activities. To construct a stable cluster, link stability is used to refine the clustering operation.

As stated earlier, the CUs located in the same PU interference area would share a same set of available channels. Thus, we use the residual existing time (RET) to denote the time until a GWC moves out of its current PU interference area. Therefore, the distance between GWC and PU should be monitored. At a certain time instance, let $\left(x_{i}, y_{i}\right)$ denote the Cartesian coordinates of a CU $i$, moving with velocity $\left(v_{i}, \theta_{i}\right)$. Let $\left(x_{k}, y_{k}\right)$ denote the Cartesian coordinates of a PU $k$. Let $R_{k}$ denote the maximum interference range of the PU $k$. Finally, $R E T_{i k}$ can be computed as in Eq. (1).

$$
R E T_{i k}=\frac{-(a b+c d)+\sqrt{\left(a^{2}+c^{2}\right) R_{k}^{2}-(a d-b c)^{2}}}{a^{2}+c^{2}}
$$

where $a=v_{i} \cos \theta_{i}, b=x_{i}-x_{k}, c=v_{i} \sin \theta_{i}, d=y_{i}-y_{k}$.

Intuitively the larger the value of $R E T_{i k}$, the longer the CU $i$ will stay in the interference range of PU $k$.

We assume that CUs transmit with maximal power and the wireless transmission range is fixed with a value $R$, while it is easy to extend the proposed approach to diverse transmission range scenario. The inter-node distance is dynamic as nodes move. We will predict a residual connection time $\left(R C T_{i j}\right)$ that $\mathrm{CU} i$ and $\mathrm{CU} j$ stay in each other's transmission range. A lot of prediction algorithms have been proposed [29-31]. A prediction algorithm similar to that 
in [29] is adopted in this paper. For the prediction detail, please refer to the paper [29]. With RET and RCT, we use the residual available time, $R T_{i j}$, to denote link stability as

$$
R T_{i j}=\min \left\{R C T_{i j}, \max _{k \in\left\{\mathbb{p}_{i j}\right\}}\left\{\min \left\{R E T_{i, k}, R E T_{j, k}\right\}\right\}\right\}
$$

where $i, j$ are the two ends of a link and $\left\{\mathbb{P}_{i j}\right\}=\left\{\mathbb{p}_{i}\right\} \cap\left\{\mathbb{p}_{j}\right\}$.

With the value of link stability, the next step is to cluster them using link stability. A pair of GWCs, whose inter-link stability is greater than a threshold $R T_{t h}$, forms a new sub-cluster or joins an existing one (i.e., if one of the GWCs is already a member of a sub-cluster). It means that any GWC joined into a cluster is connected with at least one other GWC. Therefore, all GWCs in the same cluster are connected.

\subsection{Defining the cluster head and the TTL value of a cluster}

After the clustering operation, the next step is to determine the Cluster Head $(\mathrm{CH})$. A CH initiates communication and controls the flow of signaling messages within its cluster. It is required that information on $\mathrm{CH}$ can be communicated to each GWC within its corresponding cluster. Since all GWCs in a cluster are connected, all GWCs satisfy this requirement. As described above, frequent $\mathrm{CH}$ change would waste large amounts of scarce network resources and result in low end-to-end performance. This paper aims to select a more stable $\mathrm{CH}$ and decide a more stable path from the source nodes to the $\mathrm{CH}$. In this section, we propose two algorithms: one for selecting the $\mathrm{CH}$ and other for determining the TTL value and the path to $\mathrm{CH}$. The algorithms are given as follows.

(1) $\mathrm{CH}$ selection among all candidate GWCs:

(a) For each candidate GWC, collect its entire neighbor GWCs and calculate the link stability values of all links with its neighbor GWCs according to Eq. (2).

(b) Calculate the sum of the link stability values.

(c) Select the GWC with the highest sum value as the $\mathrm{CH}$.

(2) TTL value determination:

(a) Define the link weight as the link stability, and the path weight as the minimum link weight of all links along the path to the $\mathrm{CH}$.

(b) Set the path weights to infinity for the $\mathrm{CH}$ and to zero for neighbors. Mark all the neighbors as unvisited and the $\mathrm{CH}$ as the current one.

(c) Calculate the path weights from the $\mathrm{CH}$ to unvisited neighbors via the current node. If they are larger than the previously recorded ones, update the path weights to them.

(d) Mark the current node as visited, and set the unvisited node with largest path weight as the current node. Then, repeat from (c) until all the GWCs in the cluster are visited.

(e) Denote the largest hop number of all paths developed as the TTL value.

By running the above algorithms, the stable links will be used (or the links with low stability will be avoided) in the resulting path to the $\mathrm{CH}$, with which a more stable cluster topology. And the stable cluster topology serves as a stable backbone for the ordinary CUs to the cloud. The time complexity of our clustering algorithm is $\mathrm{O}\left(\mathrm{n}^{2}\right)$, where $\mathrm{n}$ is the number of mobile gateway candidates.

\section{Mobile Gateway Management Mechanism}

Having performed the clustering of GWCs in the considered architecture, we now focus on the gateway selection for each source CU to connect to the cloud. We design a mechanism called Mobile Gateway Management, which consists of two major operations: 1) gateway discovery 
and advertisement and 2) mobile gateway selection. The gateway discovery and advertisement operation is launched to discover gateway for ordinary CUs. The gateway selection mechanism is initiated to select a better gateway when a source wants to communicate with the cloud.

\subsection{Hybrid Gateway discovery/advertisement}

The traditional gateway discovery mechanisms are proactive or reactive in nature. In this paper, a hybrid one is adopted, which combines the advantages of both approaches. The Gateway Discovery/Advertisement mechanism is shown as follows.

(1) The CH sends periodic Cluster Advertisement (CA) within the cluster using the TTL value (as discussed in Section 3.3), which determines the cluster advertisement zone. The TTL value promises that all GWCs belonging to a cluster could receive CA messages from the $\mathrm{CH}$. Accordingly, every GWC knows information about its $\mathrm{CH}$ from the CA messages. (2) A gateway that receives periodic $\mathrm{CA}$ messages would relay to its $\mathrm{CH}$ with Gateway Acknowledgement (GA). Accordingly, the $\mathrm{CH}$ could update the information of all the GWC in its cluster. And the ordinary CUs, who receive the GA message, could get informed about the GWC and update the record of the GWC in its GWC table.

(3) When an ordinary CU desires to access the cloud via a gateway, it first checks whether there is any GWC record in its GWC table.

(a) If there is at least one GWC listed in the GWC table, then it will send a Gateway Solicitation (GWSOL) message to the GWC(s) on the list. The GWCs, which receive the GWSOL message, will then respond with a GA message indicating the metric information of the $\mathrm{CH}$ in the cluster.

(b) If there is no any GWC in the GWC table, then it will broadcast a Gateway Solicitation (GWSOL) message to the next-hop neighbors, until the GWSOL reaches to at least one GWC in the cluster. After that, the GWC will then respond with a GA message indicating the metric information of the $\mathrm{CH}$ in the cluster.

The advantages of this hybrid gateway discovery mechanism are twofold. First, Cluster Advertisement is limited to the GWCs in a cluster, and thus it prevents flooding the whole network. Second, it is sufficient for the GWSOL to reach all GWCs in a cluster to get information about the $\mathrm{CH}$, instead of reaching directly the $\mathrm{CH}$. From running the above gateway discovery procedures, the metric information needed for gateway selection is notified to the source CU. Gateway is then selected by the source CU using the gateway selection mechanism described in Section 4.2.

\subsection{QoS-based gateway selection algorithm}

In this section, we propose a QoS-based gateway selection algorithm, which is shown in Algorithm 1. It is employed upon the available CHs and GWCs in the cluster. The algorithm is based on the Simple Additive Weighting (SAW) technique. Each traffic from a source CU has its own QoS constraints. For example, the voice traffic is very delay sensitive. Thus, the selection of gateway should be performed based on the QoS constraints. In this paper, the considered criteria of the GWC are listed as follows. Criteria are input parameters that are either measured or received by the source in the GA message from the responding GWC. Every types of traffic has its own threshold value for each criterion.

Load of the $\mathrm{CH}$ : it is the occupation ratio of the buffering queue of the $\mathrm{CH}$.

Source-GWC-CH path weight: It is the path weight of the path through the GWC between source and the $\mathrm{CH}$, which can be computed using Eq. (2). It reflects well the stability of the 
path and its lifetime between source and the $\mathrm{CH}$.

Source-GWC-CH hop distance: It is the hop count number of the path from source to the $\mathrm{CH}$ via the GWC. It is calculated as the sum of the hop count from the source to the GWC and the hop count from GWC to the CH given in Section 3-C.

Criteria are divided into two categories: positive and negative. A positive criterion is a criterion that is considered better while increasing, such as Source-GWC-CH path weight. However, a negative criterion is a criterion that goes worse as it increases, such as Load of the $\mathrm{CH}$ and Source-GWC-CH hop distance.

\section{Begin Algorithm 1}

When receiving $G A$ message by the source:

The source vehicle will always check the three criteria $\mathrm{X}_{\mathrm{i}}(\mathrm{i}=1 \ldots 3)$ of the $\mathrm{CH}$ : Load of the $\mathrm{CH}$, Source-GWC-CH path weight, and Source-GWC-CH hop distance.

for each criteria $\mathrm{X}_{i}$ of the $\mathrm{CH}$, where $1 \leq \mathrm{i} \leq 3$, do

if $X_{i}$ do not satisfy the threshold requirement $X_{i t h}$ then

discard this GA message and wait for another GA message.

else

if $X_{i}$ is positive criteria then

$$
Y_{i}=\frac{x_{i}}{x_{i t h}}
$$

else $X_{i}$ is negative criteria then

$$
Y_{i}=\frac{X_{i t h}}{X_{i}}
$$

end if

end if

end for

The source calculates the weight of each $\mathrm{CH}$ by

$$
W_{C H}=\sum_{i=1}^{3} \omega_{i} Y_{i} .
$$

The source determines the $\mathrm{CH}$ with the maximum Weight and selects it as the GATEWAY.

\section{End Algorithm 1}

\section{Algorithm 1: QoS-based gateway selection algorithm.}

It should be noted that, the above QoS-based gateway selection algorithm could select the better gateway satisfying the threshold requirements, and also the stable path from source to the $\mathrm{CH}$. The time complexity of our QoS-based gateway selection algorithm is dependent on the complexity of calculating the three metrics and the for loop. The time complexity of calculating Load of the $\mathrm{CH}$ is $\mathrm{O}(1)$. The time complexity of calculating Source-GWC-CH path weight and Source-GWC-CH hop distance is determined by the time complexity of our clustering algorithm. As described in section 3.3, the time complexity of our clustering algorithm is $\mathrm{O}\left(\mathrm{n}^{2}\right)$. The body of the For loop can be implemented to run in time $\mathrm{O}(1)$. Thus, the total time complexity of our gateway selection algorithm is $\mathrm{O}\left(\mathrm{n}^{2}\right)$. 


\section{Performance Evaluation}

In this section, we implement simulations to evaluate the performance of our proposed clustering-based mobile gateway management scheme (CGM), including the multi-metric clustering (MMC) algorithm and QoS-based gateway selection algorithm (QGWS).

\subsection{Simulation scenario}

In the simulation environment, PUs and CUs are randomly deployed in a $500 \mathrm{~m} \times 500 \mathrm{~m}$ 2-dimension square. The number of PUs is set to be 20 and the number of CUs is set to be 60 . Each PU has a licensed spectrum channel and hence there are exactly 20 channels potentially available to the CUs. Each PU could behave in state of ON (active on the channel) or OFF (inactive on the channel). We assume that the sojourn times of the PU in ON state and OFF state are random variables, which follow the exponential distributions with parameters $\lambda$ and $\mu$, respectively. The interference range of each PU is $300 \mathrm{~m}$, and the transmission range of each $\mathrm{CU}$ is $150 \mathrm{~m}$. CUs are moving in the network according to the random-walk-based mobility model, where a CU moves with a direction uniformly and a speed uniformly from 0 to $\mathrm{v}_{\max }$ with exponentially distributed epochs. CUs are equipped with a GPS receiver. Thus, each CU knows its own position and velocity. Among these CUs, some satisfying the gateway criteria are GWCs. And the density of GWCs is defined as the ratio of the number of GWCs to the total number of CUs. In the simulation, we set the density of GWCs to be $10 \%$.

To evaluate the performance of our CGM scheme, we perform comparison with existing GSM[32] and two modified version of CGM, respectively named as CGM1 and CGM2. In our CGM, we propose multi-metric clustering algorithm (MMC) and QoS-based gateway selection algorithm (QGWS) in integrated CRAHN-Cloud architecture. In CGM1, clustering is done using the Connected-dominating-set algorithm (CDS) introduced by [28] and gateway selection is done using our QGWS. In CGM2, the clustering is done using our MMC while gateway selection is done using the default gateway selection algorithm (DGWS) introduced by [33].

The performance of our CGM scheme in the integrated architecture is evaluated using the following metrics:

Average Throughput: it is the average rate of successfully transmitted data packets over the communication channel.

Packet Drop Fraction: it is the ratio of the number of unsuccessfully transmitted packets (i.e., as a result of packet drops) to the total number of packets sent from the sources.

End-to-end delay: it refers to the total time elapsed since the broadcasting of GWSOL by a source till the packet is successfully transmitted to the selected gateway.

\subsection{Impact of PU activities}

Notice that $1 / \lambda$ and $1 / \mu$ are the expected sojourn times of PU being in ON status and OFF status, respectively. $P_{o n}=\mu / \mu+\lambda$ and $P_{o f f}=\lambda / \mu+\lambda$ are the probabilities that the PU is in ON status and OFF status in an instant of time, respectively. In this section, we evaluate the impact of the PU activities upon the performances of our scheme by increasing $P_{o n}$. 


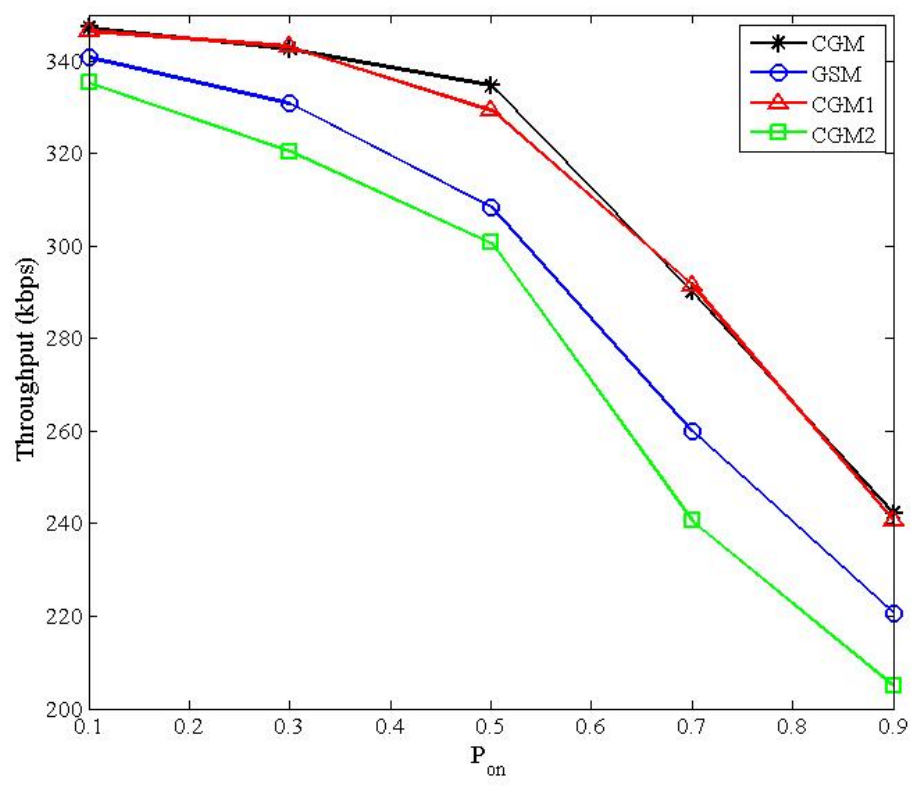

Fig. 2. Average throughput versus the ON probability of PU

Fig. 2 shows the effects of increasing the value of $P_{o n}$ on average throughput. As shown in the figure, the average throughput decreases as the value of $P_{o n}$ increases. The reason of decrease in average throughput is that available spectrum channels for the CUs to use would decrease as $P_{o n}$ increases. Hence, the communication opportunities for the CUs would be reduced and the average throughput would decrease relevantly. When the value of $P_{\text {on }}$ increases to a certain threshold, the collision among CUs would also increase the probability of transmission failure. And thus the average throughput would decrease much faster. We can also find that the average throughput of our CGM is much higher than that of GSM. As described earlier, clustering and gateway selection of GSM try to maximize the common channels among CUs. While our CGM consider both the common channels among CUs and the mobility of CUs, thus in a mobile cognitive ad hoc network, the cluster formed by our scheme would be more stable and the gateway selected would also be stable. Thus, the average throughput could be improved by using our scheme. And the improvement could reach at about $11 \%$ as $P_{\text {on }}$ increases. CGM also outperforms CGM2 in terms of average throughput. However, CGM performance is comparable with CGM1. The difference betweeen CGM1 and CGM is that CGM1 use CDS scheme for clustering, while the difference betweeen CGM2 and CGM is that CGM2 use DGWS scheme for gateway selection. And CDS clustering considers the impact of PU activities on cluster stability. And in this simulation, the CUs move slowly. Therefore, the clusters formed by both clustering algorithms are stable. Since CGM1 uses our QGWS algorithm to select gateway, the selected gateway is also more efficient and the path is also more stable. Thus, the performance of CGM1 is comparable with CGM. But CGM2 use DGWS to select gateway, with which all packets to the cloud are through cluster head. The cluster head will become the bottleneck. And it is easy to be affected by the available spectrum channels caused by PU activities. 


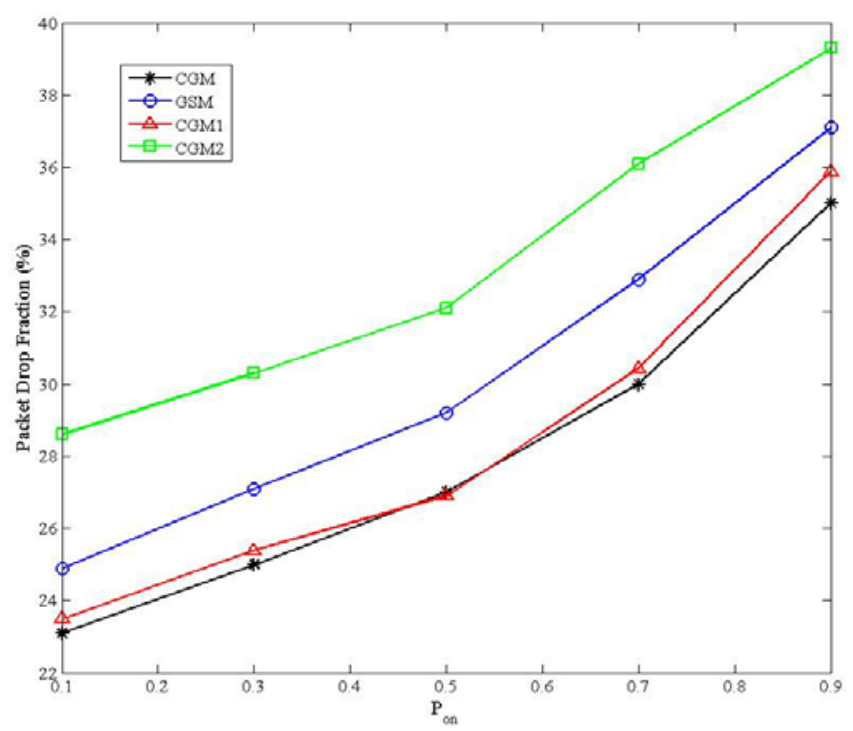

Fig. 3. Packet drop fraction versus the ON probability of PU

Fig. 3 shows the average packet drop fraction versus $P_{o n}$. As shown in the figure, the packet drop fraction increases as the value of $P_{o n}$ increases. The reason is that the probability of CU transmission being affected increases as $P_{\text {on }}$ increases. The figure shows that our CGM scheme provides better results as compared with GSM. This is due to the fact that the cluster formed is more stable and the selected gateway has lower load and more stable routing path, leading to better performance. Our CGM scheme produces packet drop fraction which is about 8\% lower than GSM. CGM also outperforms CGM2 in terms of packet drop fraction. However, CGM performance is comparable with CGM1. The reason has been addressed earlier. Both clustering algorithms consider the impact of the PU activities while clustering the network. And the CU movement is relativley slow in the simulaiton enviorment. Thus, the clusters formed by both clustering algorithms are stable. Since CGM1 uses our QGWS algorithm to select gateway, the selected gateway and the rouitng path to the selected gateway are more stable. Thus, the performance of CGM1 is comparable with CGM. But CGM2 use DGWS to select gateway, with which all packets to the cloud are through cluster head. It is easy to be affected by the available spectrum channels caused by PU activities. 


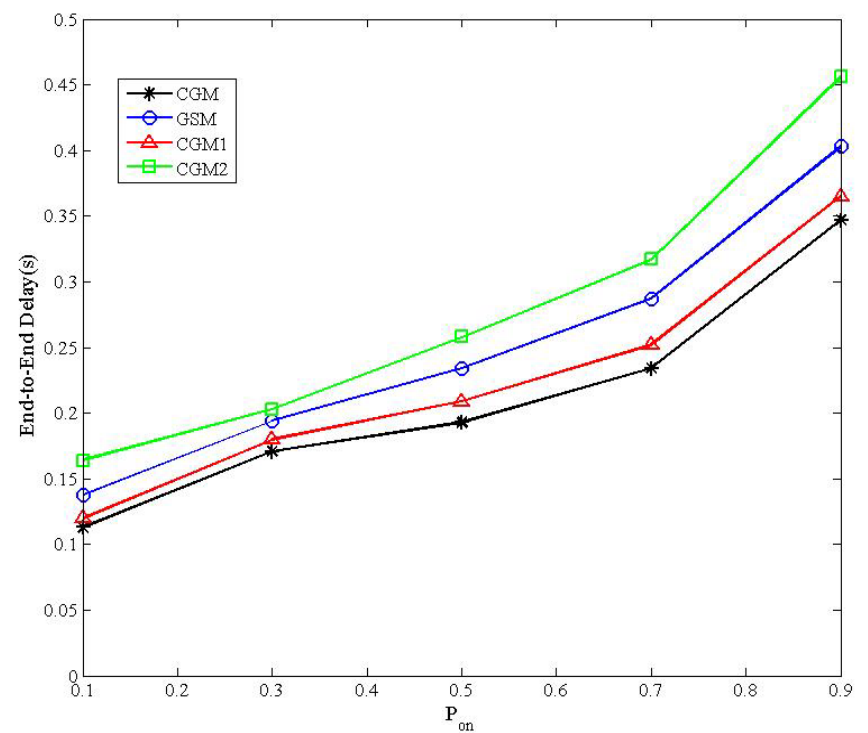

Fig. 4. Average end-to-end delay versus the ON probability of PU

Fig. 4 shows the average end-to-end delay versus $P_{o n}$. Again, the figure shows that CGM outperforms GSM and CGM2 in terms of average end-to-end delay. The reasons are similar as described earlier for average throughput and packet drop fraction. And the average end-to-end delay achieved by our CGM scheme is about $10 \%$ lower than that achieved by GSM. But we can see that the end-to-end delay achieved by CGM1 is slightly longer comparable with that achieved by CGM. Since clustering in CGM1 does not consider the effect of CU mobility and gateway selection takes it into account, it would choose a longer path to the gateway to avoid the unstable link due to CU mobility. Therefore, the average end-to-end delay is slightly longer.

\subsection{Impact of CU velocity}

Here, we evaluate the impact of the CU velocity upon the performances of the schemes.

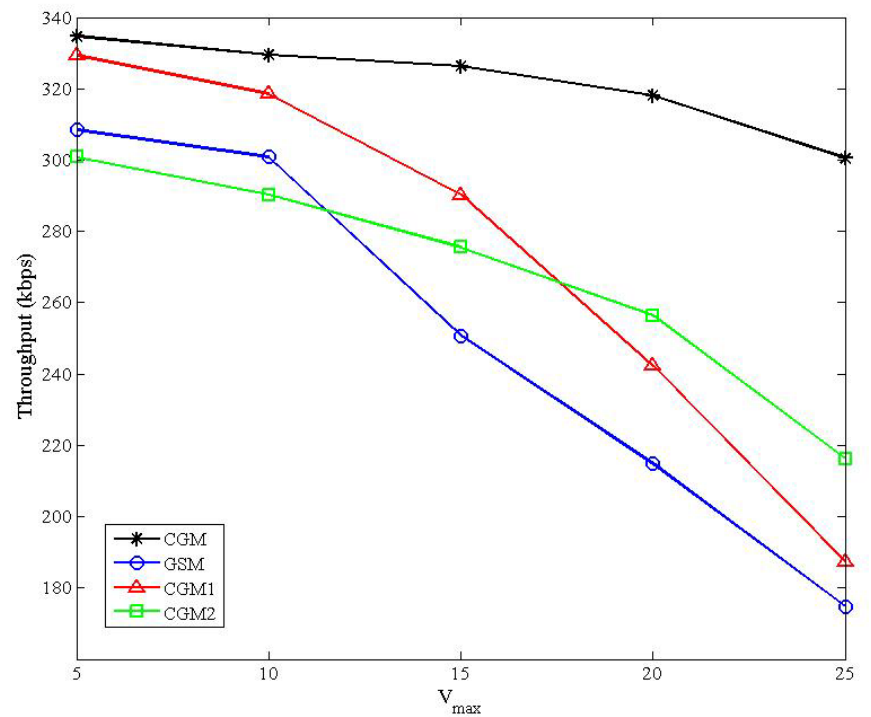

Fig. 5. Average throughput versus the maximum velocity of CU 
Fig. 5 shows the average throughput while the maximum velocity of CU increases. As shown in the figure, the average throughput is decreased as $v_{\max }$ increases. When $\mathrm{v}_{\max }$ increases, the link becomes unstable, and the probability that the selected gateway is not available increases, and thus the average throughput decreases. But it is shown that the average throughput achieved by our CGM scheme decreases much slower compared with the other three schemes. It is because our clustering algorithm considers CU velocity as one of the clustering criteria and it will develop more stable backbone for the CUs. Since CGM2 also use the same clustering algorithm, the achieved average throughput decreased also slower as compared with GSM and CGM1. The average throughput improvement of our CGM scheme is over $50 \%$ compared with GSM, as $v_{\max }$ achieves 20 .

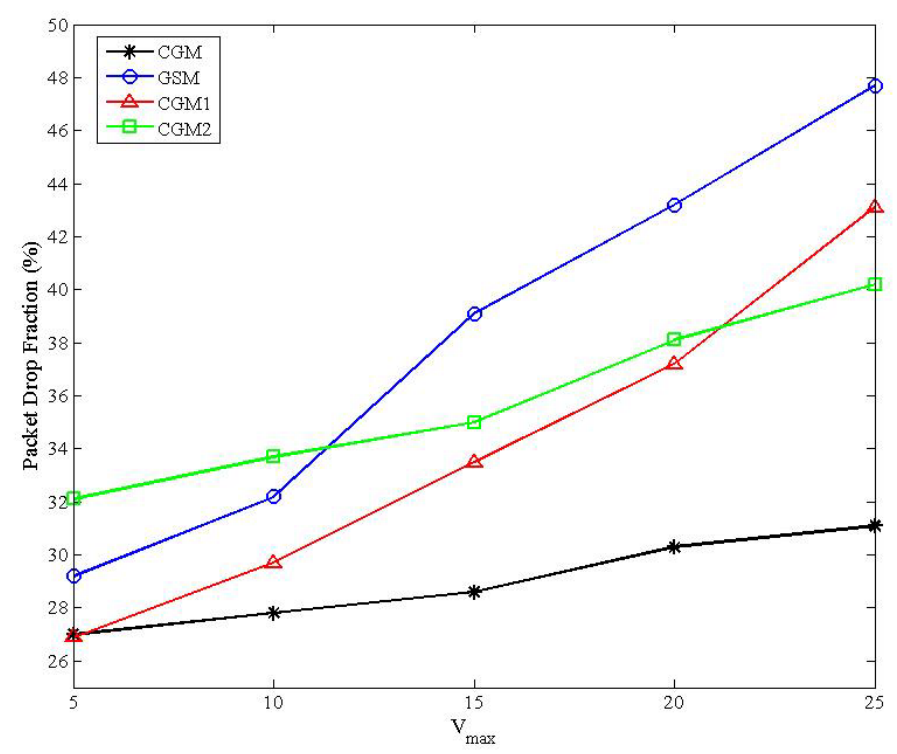

Fig. 6. Packet drop fraction versus the maximum velocity of CU

Fig. 6 shows the packet drop fraction versus the maximum velocity of CU. The figure shows better results of our CGM scheme as compared with the other three schemes. And our CGM scheme could produce at most 30\% lower packet drop fraction than the GSM scheme. This is due to the fact that both the clustering and gateway selection processes of CGM take CU mobility into account. In fact, as the maximum velocity increases, the link stability decrease. Therefore, the links become unstable. However, with our algorithm, more stable backbone and higher path stability can be achieved. The transmission failure due to CU mobility could be reduced, and thus the packet drop fraction could also be reduced. 


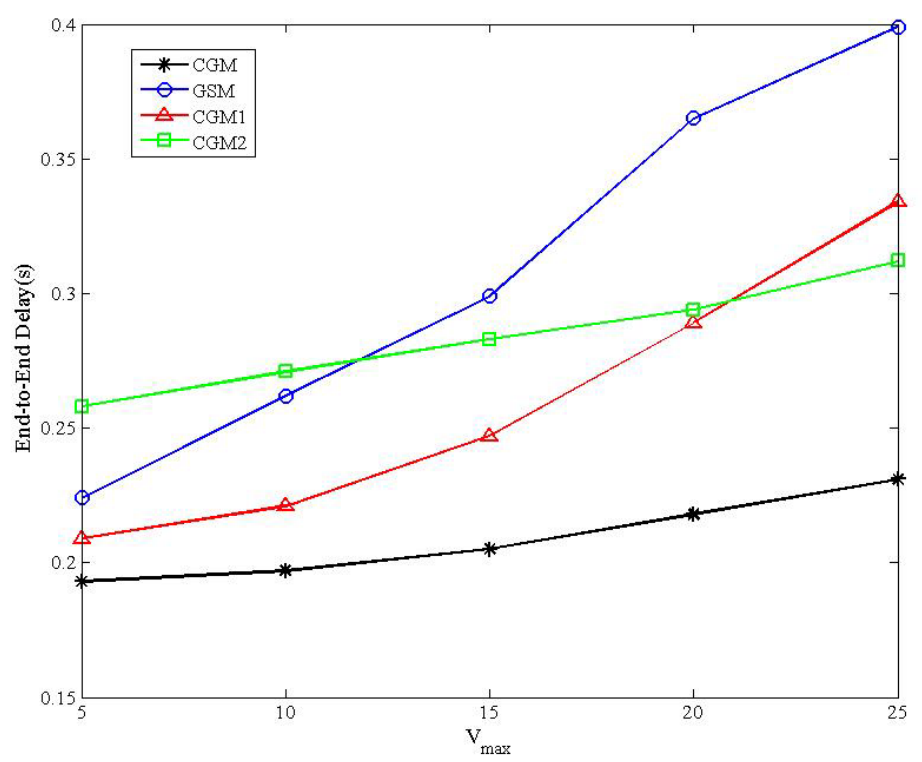

Fig. 7. Average end-to-end delay versus the maximum velocity of CU

Fig. 7 shows the impact of the maximum velocity of CU on the average end-to-end delay. From Fig. 7, it can be seen that the average end-to-end delay also increases as $v_{\max }$ increases. When $\mathrm{v}_{\max }$ increases, the link becomes unstable, and the probability that the transmission failure increases, and thus the average end-to-end delay increases. However, the end-to-end delay of our CGM scheme increases much slower as compared with the other three schemes. Since the CU mobility is considered in the clustering and gateway selection processes, re-clustering and path failure due to CU mobility can be reduced, which in turn causes less end-to-end delay.

\section{Conclusion}

In this paper, a novel architecture is introduced to integrate the cloud with CRAHNs. In this architecture, a number of gateways are selected to connect ordinary CUs with the cloud. PU interference, link stability, and load of the $\mathrm{CH}$ are taken into consideration when clustering and selecting gateway candidates. Gateway discovery scenarios are also considered and a hybrid gateway discovery/advertisement scheme is presented. A QoS-based gateway selection algorithm is proposed to select the optimal gateway for each source CU. The performance of the overall architecture was evaluated using computer simulations. Simulation results show that our scheme performs better in terms of throughput, packet delivery fraction, and end-to-end delay even when the ON probability of PU and the velocity of CUs increase.

\section{References}

[1] J. Mitola and G. Maguire, "Cognitive radio: making software radios more personal," IEEE Personal Communications, Vol. 6, No. 4, pp. 13-18, 1999. Article (CrossRef Link)

[2] S. Haykin, “Cognitive radio: Brain-empowered wireless communications," IEEE Journal on Selected Areas in Communications, vol. 23, no. 2, pp. 201-220, Feb. 2005. Article (CrossRef Link) 
[3] Ian F. Akyildiz, W. Lee, M. Vuran, and S. Mohanty, "NeXt generation/dynamic spectrum access/cognitive radio wireless networks: A survey,” Computer Networks, vol. 50, no. 13, pp. 2127-2159, 2006. Article (CrossRef Link)

[4] Elias Z. Tragos, Sherali Zeadally, Alexandros G. Fragkiadakis and Vasilios A. Siris, "Spectrum Assignment in Cognitive Radio Networks: A Comprehensive Survey,” IEEE Communications Surveys \& Tutorials, Vol. 15, Iss. 3, pp. 1108-1135, 2013. Article (CrossRef Link)

[5] N. Devroye, M. Vu, and V. Tarokh, “Cognitive radio networks,” IEEE Signal Process. Mag., vol. 25, no. 6, pp. 12-23, Nov. 2008. Article (CrossRef Link)

[6] I. F. Akyildiz, W. Y. Lee, and K. R. Chowdhury, "CRAHNs: Cognitive radio ad hoc networks," Ad Hoc Networks Elsevier, 7(5), pp. 810-836, 2009. Article (CrossRef Link)

[7] Kamal Deep Singh, Priyanka Rawat and Jean-Marie Bonnin, "Cognitive radio for vehicular ad hoc networks (CR-VANETs): approaches and challenges," EURASIP Journal on Wireless Communications and Networking, no. 49, 2014. Article (CrossRef Link)

[8] R. Yu, Y. Zhang, S. Gjessing, W. Xia, K. Yang, "Toward Cloud-Based Vehicular Networks with Efficient Resource Management,” IEEE Network, vol. 27, no. 5, pp. 48-55, Sep 2013.

Article (CrossRef Link)

[9] Feng Ge, Heshan Lin, Amin Khajeh, C. Jason Chiang, Ahmed M. Eltawil, Charles W. Bostian, Wu-chun Feng, and Ritu Chadha, "Cognitive radio rides on the cloud," in Proc. of Military Communications Conference (MILCOM), pp.1448-1453, 2011. Article (CrossRef Link)

[10] Y. B Reddy and S. Ellis, "Modeling Cognitive Radio Networks for Efficient Data Transfer Using Cloud Link," in Proc. of Tenth International Conference on Information Technology: New Generations (ITNG), pp. 525-530, 2013. Article (CrossRef Link)

[11] C.H. Ko, D.H. T. Huang and S.H. Wu, "Cooperative Spectrum Sensing in TV White Spaces: When Cognitive Radio Meets Cloud," in Proc. of IEEE Conference on Computer Communications Workshops (INFOCOM WKSHPS), pp.672-677, 2011. Article (CrossRef Link)

[12] Sau-Hsuan Wu, Hsi-Lu Chao, Chun-Hsien Ko, Shang-Ru Mo, et al, “A Cloud Model and Concept Prototype for Cognitive Radio Networks,” IEEE Wireless Communications, vol.19, iss.4, pp. 49-58, 2012. Article (CrossRef Link)

[13] N. Cordeshi, D. Amendola, and E. Baccarelli, "Reliable adaptive resource management for cognitive cloud vehicular networks,” IEEE Transactions on Vehicular Technology, vol. 64, no. 6, pp. 2528-2537, Jun. 2015. Article (CrossRef Link)

[14] H.T. Dinh, C. Lee, D. Niyato, and P. Wang, "A survey of mobile cloud computing: architecture, applications, and approaches,” Wireless Communications and Mobile Computing, 2011. Article (CrossRef Link)

[15] P. Thanapal, A. Varshney, and M. A. Saleem Durai, “A Survey on Cloud Computing for Mobile Users: Making Smartphones Last Longer with Computation Offload,” International Journal of Computer Applications, vol. 56, no.18, 2012.

[16] Pablo Punal Pereira, Jens Eliasson, Rumen Kyusakov, Jerker Delsing, Asma Raayatinezhad, and Mia Johansson, "Enabling Cloud Connectivity for Mobile Internet of Things Applications," in Proc. of IEEE Seventh International Symposium on Service-Oriented System Engineering (SOSE’13), pp. 518-526, 2013. Article (CrossRef Link)

[17] M. Jo, T. Maksymyuk, B. Strykhalyuk, and C.-H. Cho, "Deviceto-device-based heterogeneous radio access network architecture for mobile cloud computing,” IEEE Wireless Commun., vol. 22, no. 3, pp. 50-58, Mar. 2015. Article (CrossRef Link)

[18] Vinod Namboodiri, Manish Agarwal , and Lixin Gao, "A study on the feasibility of mobile gateways for vehicular ad-hoc networks," in Proc. of Proceedings of the 1st ACM international workshop on Vehicular ad hoc networks, October 01-01, 2004, Philadelphia, PA, USA. Article (CrossRef Link)

[19] A. Benslimane, T. Taleb, R. Sivaraj, "Dynamic Clustering-Based Adaptive Mobile Gateway Management in Integrated VANET C 3G Heterogeneous Wireless Networks,” IEEE Journal on Selected Areas in Communication, vol. 29, no. 3, pp. 559-570, March 2011.

Article (CrossRef Link) 
[20] P. Angove, M. O'Grady, J. Hayes, B. O'Flynn, G. M. P. O'Hare, and D. Diamond, “A Mobile Gateway for Remote Interaction With Wireless Sensor Networks,” Sensors Journal IEEE, pp. 3309-3310, Dec. 2011. Article (CrossRef Link)

[21] H. Ammari, and H. El-Rewini, "Integration of Mobile Ad Hoc Networks and the Internet Using Mobile Gateways," in Proc. of Proceedings of the 18th International Parallel and Distributed Processing Symposium, pp. 218-225, April 2004. Article (CrossRef Link)

[22] T. Chen, H. Zhang, G. M. Maggio, and I. Chlamtac, "CogMesh: A cluster-based cognitive radio network," in Proc. of 2nd IEEE International Symposium on New Frontier in Dynamic Spectrum Access Networks, pp. 168-178, 2007. Article (CrossRef Link)

[23] C. Sun, W. Zhang, and K. Ben, "Cluster-based cooperative spectrum sensing in cognitive radio systems,” in Proc. of IEEE International Conference on Communications (ICC'07), pp. 2511-2515, Jun. 2007. Article (CrossRef Link)

[24] S. Liu, L. Lazos, and M. Krunz, "Cluster-Based Control Channel Allocation in Opportunistic Cognitive Radio Networks,” IEEE Transactions on Mobile Computing, vol. 11, no. 10, pp. 1436-1449, 2012. Article (CrossRef Link)

[25] H. Zhang, Z. Zhang, H. Dai, R. Yin, and X. Chen, "Distributed spectrum-aware clustering in cognitive radio sensor networks," in Proc. of Global Telecommunications Conference (GLOBECOM 2011), pp. 1-6, 2011. Article (CrossRef Link)

[26] D. Li and J. Gross, "Robust clustering of ad-hoc cognitive radio networks under opportunistic spectrum access," in Proc. of IEEE International Conference on Communications (ICC 2011), pp. 1-6, 2011. Article (CrossRef Link)

[27] L. Lazos, S. Liu, and M. Krunz, "Spectrum opportunity-based control channel assignment in cognitive radio networks," in Proc. of 6th Annual IEEE Communications Society Conference on Sensor, Mesh and Ad Hoc Communications and Networks (SECON’09), pp. 1-9, 2009. Article (CrossRef Link)

[28] Zhiyong Lin, Hai Liu, Xiaowen Chu, Yiu-Wing Leung and Ivan Stojmenovic, “Constructing Connected-Dominating-Set with Maximum Lifetime in Cognitive Radio Networks," IEEE Transactions on Computers, vol. pp, iss. 99, 2013. Article (CrossRef Link)

[29] Y. Bai, and L. Gong, "Link availability prediction with radio irregularity coverage for mobile multi-hop networks,” IEEE Communications Letters, vol. 14, no. 6, pp. 518-520, 2010. Article (CrossRef Link)

[30] S. Jiang, “An enhanced prediction-based link availability estimation for MANETs,” IEEE Transactions on Communications, vol. 52, no. 2, pp. 183-186, Feb. 2004. Article (CrossRef Link)

[31] Q. Guan, F. R. Yu, S. Jiang and G. Wei, "Prediction-based topology control and routing in cognitive radio mobile ad hoc networks," IEEE Transactions on Vehicular Technology, vol. 59, no. 9, pp.4443 -4452, 2010. Article (CrossRef Link)

[32] N. ul Hasan, W. Ejaz, K. Manzoor, and H. S. Kim, "GSM: gateway selection mechanism for strengthening inter-cluster coordination in cognitive radio ad hoc networks," EURASIP Journal on Wireless Communications and Networking, vol. 2013, no. 1, pp. 1-11, Jun. 2013. Article (CrossRef Link)

[33] G. Zhioua, H. Labiod, N. Tabbane, S. Tabbane, "FQGwS: A Gateway Selection Algorithm in a Hybrid Clustered VANET LTE-Advanced Network: Complexity and Performances,” in Proc. of Proceeding of 2014 International Conference on Computing Networking and Communications (ICNC), pp. 413-417, 2014. Article (CrossRef Link) 


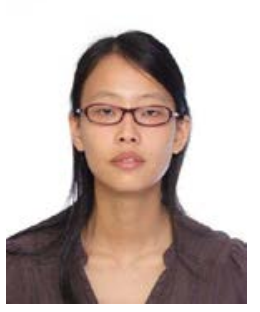

Ling HOU received her B.Eng. degree from Beijing University of Posts and Telecommunications and her Ph.D degree from City University of Hong Kong. After that, she worked as research assistant in City Uniersity of Hong Kong and the Open University of Hong Kong. Her research interests include cognitive radio networks, cloud computing, and network science.

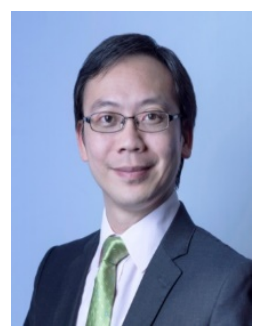

Angus K. Y. Wong received his B.Sc. and Ph.D degrees in Information Technology from City University of Hong Kong. Since he began his academic career in Macao Polytechnic Institute, he has constantly carried out active research work and provided consultancy services. After serving the institute for 10 years, he returned to Hong Kong and joined the School of Science and Technology at the Open University of Hong Kong in 2013 as an Associate Professor and the Programme Leader of Engineering Sciences. His research interests include Internet systems, network infrastructure security, cognitive radio networks, and network science.

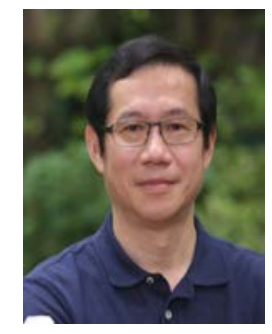

K .H. Yeung is an associate professor in the Department of Electronic Engineering, City University of Hong Kong, as well as a consultant in computer networking and communication systems for government and corporate clients. His research interests include networking infrastructure security, mobile communications systems, and Internet systems. Yeung has a PhD in Information Engineering from The Chinese University of Hong Kong.

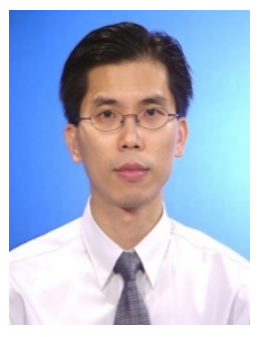

Sheung-On Choy received his B.Eng. and Ph.D degrees in electronic and information engineering from the Hong Kong Polytechnic University. He is currently an Associate Professor in the Open University of Hong Kong. His research interests include internet computing, web technologies, and cybersecurity. 